\title{
AVALIAÇÃO DA REGIÃO CORPÓREA E DOSE DA TUBERCULINA NO DIAGNÓSTICO IMUNOALÉRGICO EM OVINOS (OVIS ARIES) EXPERIMENTALMENTE SENSIBILIZADOS COM INÓCULO INATIVADO DE MYCOBACTERIUM BOVIS
}

\author{
D.M.R.S. Yuri ${ }^{1}$, S.R. Pinheiro' ${ }^{2}$, E. Roxo ${ }^{3}$, P.M.P.C. Mota $^{4}$, R.S. Jordão ${ }^{5}$, F.J. Benesi ${ }^{1}$
}

${ }^{1}$ Universidade de São Paulo, Faculdade de Medicina Veterinária e Zootecnia, Departamento de Clínica Médica, Av. Prof. Dr. Orlando Marques de Paiva, 87, CEP 05508-900, São Paulo, SP, Brasil. E-mail: falecomayuri@gmail.com

\section{RESUMO}

O objetivo foi a investigação do local de aplicação do derivado proteico purificado (PPD) bovino, empregado no teste imunoalérgico da tuberculose, em 15 ovinos (Ovis aries) experimentalmente sensibilizados com Mycobacterium bovis $\mathrm{AN}_{5}$. A partir da melhor resposta obtida na fase de identificação da região corpórea, avaliou-se a dose de PPD bovino a ser aplicada. Decorridos 60 dias da sensibilização, o PPD bovino foi aplicado em cinco distintas regiões corpóreas (cervical média, torácica dorsal, abdominal dorsal, prega da cauda e face medial proximal do membro pélvico face interna da coxa) com a mensuração da espessura da dobra de pele e da reação através de cutímetro de mola, nos momentos antes (0h) e após aplicação (12h, 24h, 48h, 72h e 96h). A análise dos resultados obtidos foi significante, em ordem decrescente, para as regiões: torácica dorsal, cervical média e abdominal dorsal às 48 e 72h após aplicação do PPD bovino. Elegeu-se a região torácica dorsal como melhor local de aplicação. Recomenda-se que a leitura da reação tuberculínica em ovinos deve ser efetuada às 72h da aplicação do PPD, utilizando-se a dose de 0,1 mL.

PALAVRAS-CHAVE: Tuberculose, PPD, ovino, alergoreação, zoonose.

\section{ABSTRACT}

EVALUATION OF THE BODY REGION AND TUBERCULIN DOSE IN THE DIAGNOSIS OF THE ALLERGIC RESPONSE IN SHEEP (OVIS ARIES) EXPERIMENTALLY SENSITIZED WITH INACTIVATED INOCULUM FROM Mycobacterium BOVIS. This study was aimed to investigate the best area for application of bovine purified protein derivative (PPD) for the imunoallergic test for tuberculosis, in 15 sheep (Ovis aries) experimentally sensitized with Mycobacterium bovis $\mathrm{AN}_{5}$. Based on the best response obtained in the previous phase, the dose of bovine PPD to be applied was evaluated. After 60 days of sensitization, the bovine PPD was applied at 5 distinct body regions to measure the skinfold thickness and reaction, at moments before $(0 \mathrm{~h})$ and after injection $(12 \mathrm{~h}, 24 \mathrm{~h}$, 48h, 72h and 96h), with skinfold calipers. Statistical analysis showed significance for the best site of application: the dorsal thoracic region, followed by the middle cervical and dorsal abdominal, at 48 and 72 hours after injection of bovine PPD. The thoracic region was chosen as the best site for application. It is recommended that the reading of the tuberculin response in sheep should be made $72 \mathrm{~h}$ after the PPD application, using a dose of $0.1 \mathrm{~mL}$.

KEY WORDS: Tuberculosis, PPD, sheep, allergic response, zoonosis.

\section{INTRODUÇÃO}

No período de 1998 a 2008, o crescimento da ovinoculturabrasileirafoide $16,5 \%$ eem 2008 , orebanhoovino brasileiro foi estimado em 16.628 .571 cabeças (IBGE, 2009). Esse crescimento tem sido atribuído ao aumento do consumo dos produtos de origem ovina, como queijos e carnes, pela população brasileira (VALENTE, 2009).

${ }^{2}$ Universidade de São Paulo, Faculdade de Medicina Veterinária e Zootecnia, Departamento de Medicina Veterinária Preventiva e Saúde Animal, São Paulo, SP, Brasil.

${ }^{3}$ Instituto Biológico, Centro de Pesquisa e Desenvolvimento de Sanidade Animal, São Paulo, SP, Brasil.

${ }^{4}$ Laboratório Nacional Agropecuário (LANAGRO), Pedro Leopoldo, MG, Brasil.

Instituto Biológico, Unidade Laboratorial de Referência para Produção de Imunobiológicos e Insumos para Uso Animal, São Paulo, SP, Brasil. 
A tuberculose, considerada desde a pré-história como uma doença infecciosa crônica e transmissível entre os mamíferos, destaca-se como uma das zoonoses que acometem os ruminantes domésticos (Leite; Telarolli Júnior, 1997). Proporcionalmente à grande prevalência da tuberculose nos bovinos, há ainda um pequeno número de relatos isolados de tuberculose em ovinos (M'FADYEAN, 1902; CORDES et al., 1981; Allen, 1988; MALONe et al., 2003).

Testes imunoalérgicos aplicados ao diagnóstico da tuberculose bovina e bubalina são mundialmente difundidos e aceitos, porém, o mesmo não ocorre com os pequenos ruminantes (caprinos e ovinos), onde não há um consenso dos valores de referência a serem utilizados nessas espécies animais (CORDES et al., 1981; DAVIDSON et al., 1981; JENSEN; SWIFT, 1982; MALONE et al., 2003).

CYrillo et al. (2007) padronizaram os valores da leitura do alergoteste da tuberculina em ovinos (Ovis aries) e MARCONDES (2007) encontrou correlação entre os resultados do teste imunoalérgico com os de cultivos e exames histopatológicos. Entretanto, à semelhança de outras espécies animais, algumas dúvidas persistem no relativo ao local de aplicação do teste e a dose de tuberculina. МотA (2003) e CoRRÊA (2008) determinaram a intensidade de resposta alérgica à tuberculina segundo o local de aplicação em bovinos e caprinos, respectivamente.

Diante da importância do tema, da escassez de dados nacionais sobre a tuberculose em ovinos, da necessidade de aprofundamento de conhecimento sobre aplicação das técnicas de diagnóstico pertinentes e do combate e erradicação da doença, foi delineado o presente estudo que teve o objetivo de avaliar a região corpórea de escolha e a dose de derivado proteico purificado (PPD) bovino a ser aplicada em ovinos (Ovis aries).

\section{MATERIAL E MÉTODOS}

Utilizaram-se 15 ovinos adultos hígidos, com resposta negativa ao Teste Cervical Comparativo (TCC) segundo os padrões estabelecidos por CYRILLO et al. (2007).

OPPD aviário $(0,5 \mathrm{mg} / \mathrm{mL})$ foi utilizado somente na triagem inicial dos ovinos submetidos ao TCC; nas demais etapas experimentais, foi utilizado o PPD bovino $(1,0 \mathrm{mg} / \mathrm{mL})$. Ambos PPD's foram produzidos pelo Instituto Biológico.

Imediatamente após a leitura do TCC de triagem inicial, os ovinos foram sensibilizados com $0,5 \mathrm{~mL}$ de inóculo inativado de $M$. bovis estirpe $\mathrm{AN}_{5}$, de concentração $4,0 \mathrm{mg} / \mathrm{mL}$, pela via intramuscular no membro posterior, conforme orientação do Laboratório Nacional Agropecuário - LANAGRO/MG.
Decorrido o período de dessensibilização de 60 dias entre aplicações, inferiram-se duas etapas experimentais: a primeira avaliando-se o local de aplicação do PPD bovino e, a segunda, a dose ideal a partir do local de melhor resposta imunoalérgica obtido na etapa anterior.

Inicialmente, aplicou-se 0,1 mL/dose de PPD bovino (lado esquerdo) e solução fisiológica (lado direito), em cinco distintas regiões corpóreas do mesmo animal, a partir das observações de SILVA et al. (2006), Marcondes (2007), Cyrillo et al. (2007), PinHeIro et al. (2007), BENESI et al. (2008), Almeida (2009), JAVED et al. (2010), Pignata et al. (2010) e Higino (2010) e adaptado da metodologia de Mota (2003) e CorrêA (2008): cervical média, torácica dorsal, abdominal dorsal, prega da cauda e face medial proximal do membro pélvico - face interna da coxa (Fig. 1).

Após 60 dias da primeira etapa, três grupos de quatro animais selecionados aleatoriamente receberam, respectivamente $0,05 \mathrm{~mL} ; 0,1 \mathrm{~mL}$ e $0,2 \mathrm{~mL}$ de PPD bovino (lado esquerdo) e solução fisiológica (lado direito) nolocal de melhor reação imunoalérgica. Olocal da aplicação intradérmica de PPD bovino ou solução fisiológica foi medido com cutímetro (Hauptner ${ }^{\circledR}$ ) antes (0h), e às 12h, 24h, 48h, 72h e 96h após aplicação.

Considerando a diferença entre os grupos controle e experimental significativa nos casos de " $p$ " menor que 0,05 pelo teste de Anderson-Darling, os parâmetros com comportamento normal foram comparados entre si pelo teste t-pareado e os rejeitados no teste de homocedasticidade foram comparados entre si pelo teste de Mann-Whitney. A interpretação estatística dos resultados obtidos foi efetuada com o método de ZAR (1984).

\section{RESULTADOS}

Não houve diferença estatística $(p>0,05)$ nas mensurações de espessura de pele feitas para o mesmo local de aplicação da tuberculina, entre os lados direito e esquerdo do animal, no momento $0 \mathrm{~h}$. Porém, para as $48 \mathrm{~h}$ e $72 \mathrm{~h}$ após a aplicação do PPD bovino, foi observada diferença significante $(\mathrm{p}<$ 0,05 ) indicando maior intensidade da alergoreação dos diferentes locais de aplicação em relação à pele normal (Tabela 1 e Fig. 2).

Quando os diferentes momentos foram avaliados (Tabela 2), observou-se diferença estatisticamente significante para as $48 \mathrm{~h}$ e $72 \mathrm{~h}$, indicando que houve uma maior intensidade da alergoreação nos diferentes locais de aplicação, em relação à pele normal. Baseado nos valores de " $p$ " obtidos às $48 \mathrm{~h}$ e $72 \mathrm{~h}$ (Tabela 3), da região torácica dorsal, pela diferença estatística às $72 \mathrm{~h}$ de leitura entre a região torácica dorsal com as regiões cervical média e prega da cauda (Tabela 3) e pela maior média de espessura 
da dobra de pele da região torácica dorsal (média $=$ $2,73 \mathrm{~mm}$ ) às 72h, optou-se por essa região como local de eleição para realização do teste imunoalérgico da tuberculose em ovinos.
Não houve diferença estatística entre a resposta imunoalérgica das diferentes doses de tuberculina $(0,05$ a $0,2 \mathrm{~mL})$, para os momentos $12 \mathrm{~h}, 24 \mathrm{~h}, 48 \mathrm{~h}, 72 \mathrm{~h}$ e 96 hós injeção.

Tabela 1 - Valores (mm) das médias, desvios padrão e "p" (pelo teste de normalidade) da espessura de pele obtidos com a aplicação do PPD bovino em ovinos experimentalmente sensibilizados com Mycobacterium bovis estirpe $\mathrm{AN}_{5^{\prime}}$ conforme a região e os momentos de leitura.

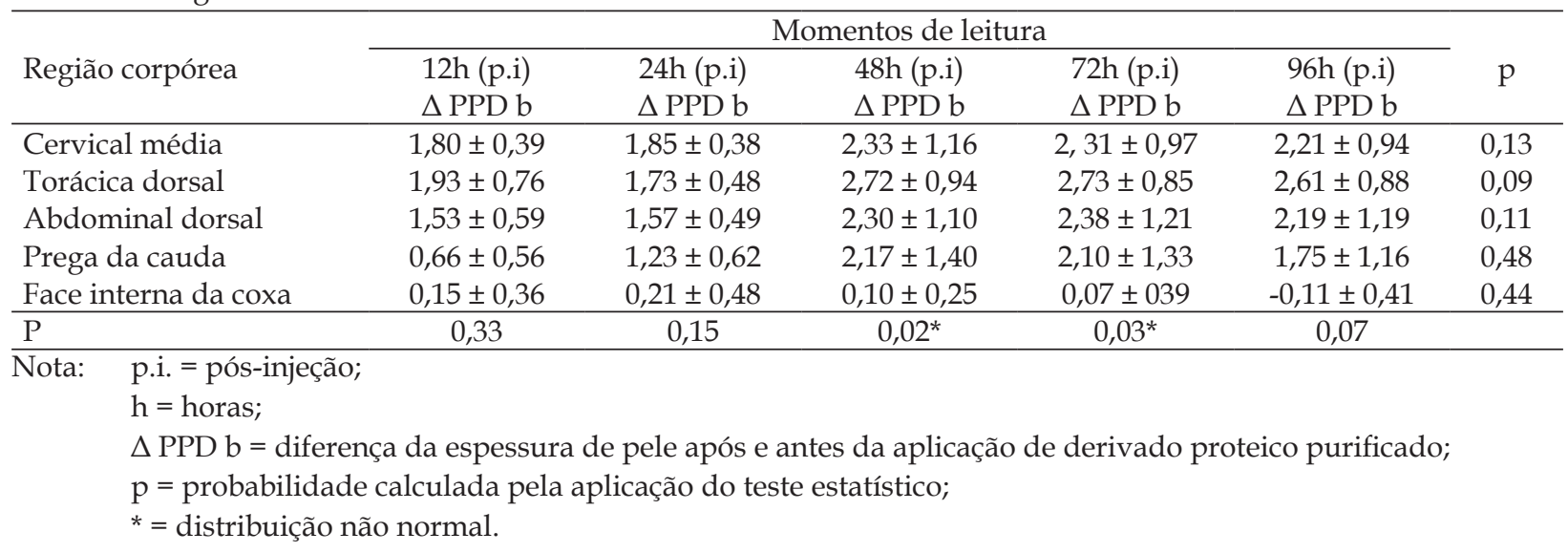

Tabela 2 - Valores de "p" (pelo teste de normalidade) do PPD bovino em ovinos experimentalmente sensibilizados com Mycobacterium bovis estirpe $\mathrm{AN}_{5}$, segundo a região e os momentos de leitura.

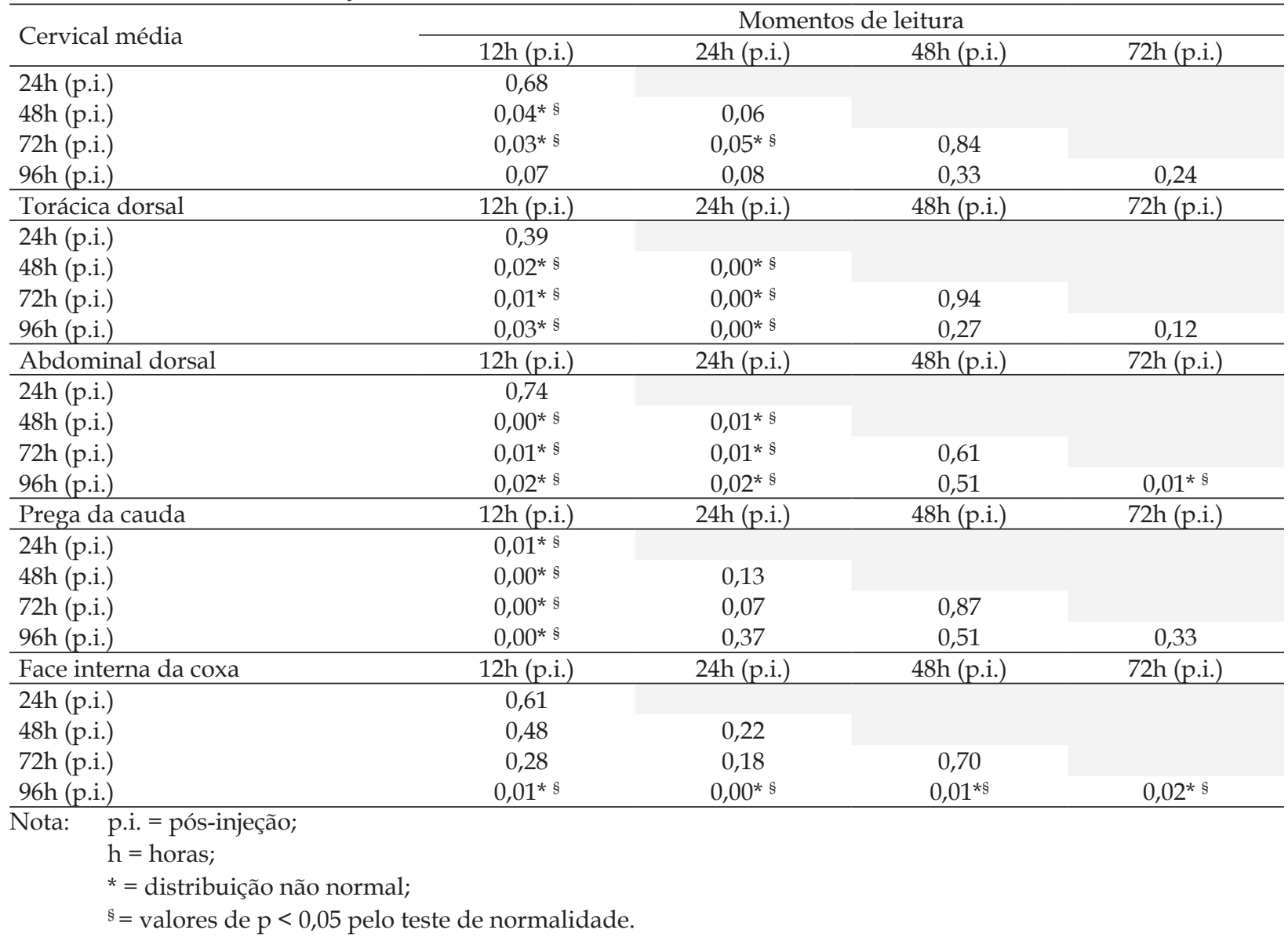


Tabela 3 - Valores de "p" (pelo teste de normalidade) obtidos através da leitura às 72 horas após aplicação do PPD bovino em ovinos experimentalmente sensibilizados com Mycobacterium bovis estirpe $\mathrm{AN}_{5}$, segundo as regiões.

\begin{tabular}{lcccc}
\hline \multirow{2}{*}{$72 \mathrm{~h}$} & \multicolumn{4}{c}{ Região corpórea } \\
\cline { 2 - 5 } & Cervical média & Torácica dorsal & Abdominal dorsal & Prega da cauda \\
\hline Torácico dorsal & $0,01^{\S}$ & & & \\
Abdomina dorsal & 0,52 & 0,06 & $0,48^{*}$ & $0,00^{*}$ \\
Prega da cauda & $0,34^{*}$ & $0,03^{*}$ & $0,00^{\S}$ & $0,00^{\S}$ \\
Face interna da coxa & $0,00^{\S}$ & &
\end{tabular}

Nota: p.i. = pós-injeção;

$\mathrm{h}=$ horas;

* = distribuição não normal (teste de Mann-Whitney);

$\S=$ valores de $\mathrm{p}<0,05$ pelo teste de normalidade.

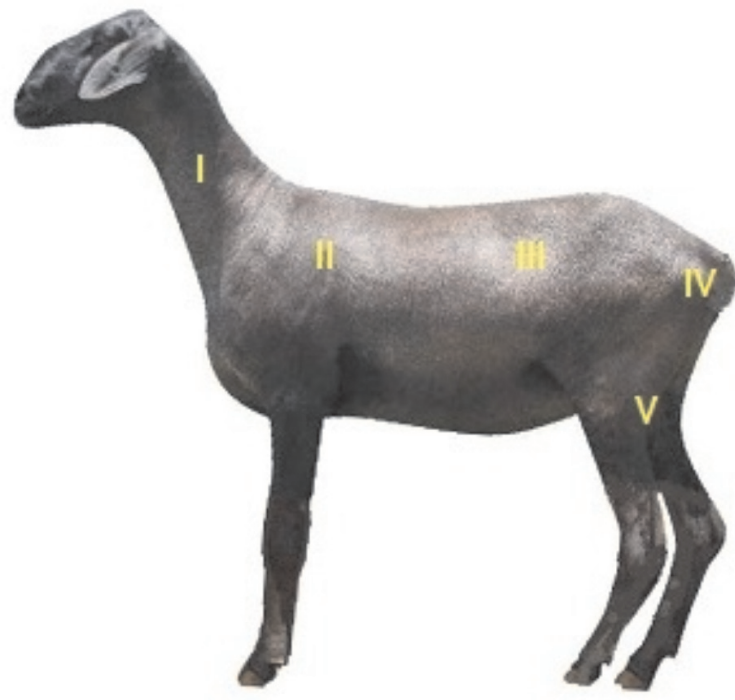

Fig. 1 - Figura esquemática assinalando as regiões da superfície corpórea do animal que foram submetidas à verificação da região corpórea ideal para tuberculinização. Região (I) cervical média, (II) torácica dorsal, (III) abdominal dorsal, (IV) prega da cauda e (V) face medial proximal do membro pélvico - face interna da coxa. Lado esquerdo: aplicação do PPD bovino; lado direito: aplicação da solução fisiológica.

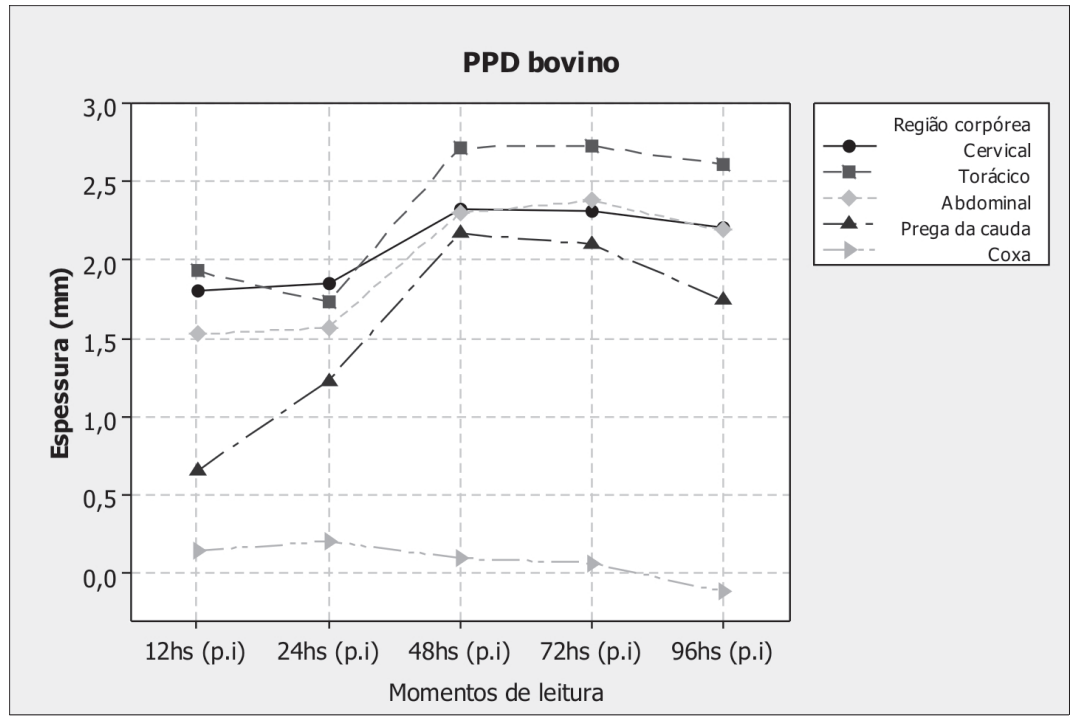

Fig. 2 - Valores (mm) médios da espessura de pele obtidos com a aplicação do PPD bovino em ovinos experimentalmente sensibilizados com Mycobacterium bovis estirpe $\mathrm{AN}_{5}$, conforme a região e os momentos de leitura.

\section{DISCUSSÃO}

Não houve diferença estatística de espessura da pele normal de ovinos entre os diferentes locais de aplicação ou entre os lados direito e esquerdo do animal, ou para o mesmo local $(\mathrm{p}>0,05)$ no momento deleitura inicial (0h). Comportamento similar foi observado com a espécie bovina (МотА, 2003) e caprina (CORRÊA, 2008). Estes dados possibilitariam a livre escolha do lado do corpo do animal para 
aplicação intradérmica da tuberculina. Entretanto, no Brasil, o Programa Nacional de Controle e Er-radicação da Brucelose e da Tuberculose (PNCEBT) (BRASIL, 2006), que vigora para as espécies bovinas e bubalinas, recomenda a aplicação do PPD no lado esquerdo do animal.

No presente estudo constataram-se que as regiões cervical, abdominal e a da prega da cauda foram as que permitiram o mais fácil acesso. A região torácica, embora de fácil acesso, apresentou dificuldade de aplicação quando o animal não era contido adequadamente, ocasionando um tensionamento da pele, prejudicando a mensuração efetuada com ocutímetro.

Em ovinos, HIEPE (1972) utilizou a região ano-caudal; CoRDEs et al. (1981) e DAVIDSON et al. (1981) aplicaram o PPD bovino na região medial da coxa. No presente trabalho, observou-se que nas regiões da prega da cauda ou face interna da coxa dos ovinos podem gerar erros na aplicação subcutânea, ao invés da intradérmica, além da dificuldade de mensuração.

Diferentemente do encontrado por HIEPE (1972), CORDEs et al. (1981) e DAVIDSON et al. (1981), que efetuaram o teste imunoalérgico, respectivamente, nas regiões ano-caudal e medial da coxa dos ovinos, no presente estudo, os locais de aplicação do PPD bovino em ovinos que apresentaram os maiores aumentos de espessura foram os das regiões torácica dorsal $(2,73 \mathrm{~mm} \pm 0,85)$, cervical média $(2,31 \mathrm{~mm} \pm$ $0,97)$ e abdominal dorsal $(2,38 \mathrm{~mm} \pm 1,21)$. Achados semelhantes foram observados por MотA (2003) e CORRÊA (2008), respectivamente em bovinos e caprinos, verificando que as regiões de maior aumento de espessura foram a cervical média em bovinos e em caprinos, as regiões abdominal e torácica dorsais e a cervical caudal.

SUTHER et al. (1974) e DOHERTY et al. (1996) constataram que em bovinos, a reação imunoalérgica apresentou maior intensidade às 48 e $72 \mathrm{~h}$ da aplicação da tuberculina, sem que houvesse distinção entre esses dois momentos, sendo analogamente observado no presente estudo.

CYRILLO et al. (2007) determinaram nos ovinos o momento de maior intensidade das reações imunoalérgicas a tuberculina, sendo-o às $48 \mathrm{~h}$ da aplicação, sem diferença do observado às $72 \mathrm{~h}$ ( \pm 6 horas). Deste modo, à semelhança de DAVIDSON et al. (1981), MALONE et al. (2003) e obedecendo às recomendações do Ministério da Agricultura, Pecuária e Abastecimento (BRASIL, 2006) para bovinos e bubalinos, o presente trabalho recomenda às $72 \mathrm{~h}$ como momento padrão de leitura do teste tuberculínico em ovinos, utilizando-se a dose de PPD bovino de 0,1 mL.

A dose de $0,2 \mathrm{~mL}$, além de representar maior consumo de antígeno, devia exigir um maior período de dessensibilização entre aplicações. Por ser pequena, a dose $0,05 \mathrm{~mL}$ poderia levar a uma dificuldade de aplicação e maior propensão a erros.

\section{CONCLUSÕES}

Nas condições de realização do referido trabalho, os resultados obtidos permitem as seguintes conclusões: todas as regiões corpóreas dos ovinos (cervical média, torácica dorsal, abdominal dorsal, prega da cauda e face medial proximal do membro pélvico - face interna da coxa) estudadas apresentaram reação imunoalérgica ao PPD bovino; o melhor local de aplicação da tuberculina é a região torácica dorsal, seguida da cervical média e abdominal dorsal. Em ovinos, as regiões da prega da cauda e da face interna da coxa não são indicadas para a realização do teste imunoalérgico da tuberculose.

Recomenda-se que a leitura da reação tuberculínica em ovinos deve ser efetuada às $72 \mathrm{~h}$ da aplicação do PPD, utilizando-se a dose de 0,1 mL.

Este experimento foi realizado conforme os princípios éticos da experimentação animal estabelecidos pela Comissão de Ética para Uso de Animais da Faculdade de Medicina Veterinária e Zootecnia da Universidade de São Paulo (n ${ }^{\circ}$ 1735/2009 de 25/11/2009).

\section{AGRADECIMENTOS}

À Coordenação de Aperfeiçoamento de Pessoal de Nível Superior (CAPES) pela concessão da bolsa. À família Dente (Descalvado, SP), por ceder gentilmente os animais ao estudo.

\section{REFERÊNCIAS}

ALLEN, G.M. Tuberculosis in sheep - a very rare disease. Surveillance, v.15, n.5, p.8-9, 1988.

ALMEIDA, C.A.S. Avaliação do Teste Cervical Comparativo no diagnóstico imunoalérgico da tuberculose em caprinos (Capra hircus). 2009. 88f. Dissertação (Mestrado em Medicina Veterinária) - Faculdade de Medicina Veterinária e Zootecnia, Universidade de São Paulo, São Paulo, 2009.

BENESI, F.J.; PINHEIRO, S.R.; MAIORKA, P.C.; SAKAMOTO, S.M.; ROXO, E.; BENITES, N.R.; BIRGEL JUNIOR, E.H.; GREGORY, L. Relato de caso: tuberculose em caprino (Capra hircus). Arquivos do Instituto Biológico, São Paulo, v.75, n.2, p.217-220, 2008.

BRASIL. Ministério da Agricultura, Pecuária e Abastecimento. Programa Nacional de Controle e Erradicação da Brucelose e da Tuberculose Animal (PNCEBT) - Manual técnico. Brasília: MAPA/DAS/DSA, 2006. 184p.

CORDES, D.O.; BULLIANS, J.A.; LAKE, D.E.; CARTER, M.E. Observations on tuberculosis caused by Mycobacterium bovis in sheep. New Zealand Veterinary Journal, v.29, n.4, p.60-62, 1981 . 
CORRÊA, J.G.Z. Estudo da resposta alérgica à tuberculina em caprinos (Capra hircus) experimentalmente sensibilizados, em diferentes regiões corpóreas. 2008. 81f. Dissertação (Mestrado em Medicina Veterinária) - Faculdade de Medicina Veterinária e Zootecnia, Universidade de São Paulo, São Paulo, 2008.

CYRILLO, F.C.; LEAL, M.L.R.; MOTTA, P.M.P.C.; SINHORINI, I.L.; VASCONCELLOS, S.A.; PINHEIRO, S.R.; BENESI, F.J. Teste de tuberculinização em ovinos (Ovis aries) experimentalmente sensibilizados. Arquivos do Instituto Biológico, São Paulo, v.74, n.3, p.191-197, 2007.

DAVIDSON, R.M.; ALLEY, M.R.; BEATSON, N.S. Tuberculosis in a flock of sheep. New Zealand Veterinary Journal, v.29, n.4, p.1-2, 1981.

DOHERTY, M.L.; BASSET, H.F.; QUINN, P.J.; DAVIS, W.C.; KELLY, A.P.; MONAGHAN, M.L. A sequential study of the bovine tuberculin reaction. Immunology, v.87, p.9-14, 1996.

HIEPE, H.T. Enfermidades de la oveja. Zaragoza: Acribia, 1972. 391p.

HIGINO, S.S.S. Isolamento de micobactérias em caprinos e ovinos abatidos no semiárido da Paraíba, Brasil. 2010. 50f. Dissertação (Mestrado em Medicina Veterinária) - Universidade Federal de Campina Grande, Patos, 2010.

IBGE - Instituto Brasileiro de Geografia e Estatística. Banco de dados agregados, 2009. Disponível em: <http:/ / www.sidra.ibge.gov.br >. Acesso em: 4 dez. 2009.

JAVED, M.T.; MUNIE, A.; SHAHID, M.; SEVERI, G.; IRFAN, M.; ARANAZ, A.; CAGIOLA, M. Percentage of reactor animals to single comparative cervical intradermal tuberculin (SCCIT) in small ruminants in Punjab Pakistan. Acta Tropical, v.113, p.88-91, 2010.

JENSEN, R.; SWIFT, B.L. Disease of sheep. 2.ed. Philadelphia: Lea \& Febiger, 1982. 330p.

LEITE, C.Q.F.; TELAROLLI JÚNIOR, R. Aspectos epidemiológicos a clínicos da tuberculose. Revista da Faculdade de Ciências Farmacêuticas, v.18, p.17-28, 1997.

M'FADYEAN, J. Tuberculosis of the sheep. Journal of Comparative Pathology and Therapeutics, v.13, p.59-60, 1902.

MALONE, F.E.; WILSON, E.C.; PLLOCK, J.M.; SKUCE, R.A. Investigation into an outuberculosereak of tuber- culosis in a flock of sheep in contact with tuberculosis cattle. Journal of Veterinary Medicine, v.50, n.10, p.500504, 2003.

MARCONDES, A.G. Micobacterioses de ovinos (Ovis aries) do Estado de São Paulo, Brasil. Correlação entre teste imunoalérgico, cultivo e histopatológico. 2007. 93f. Tese (Doutorado em Epidemiologia Experimental e Aplicada às Zoonoses) - Faculdade de Medicina Veterinária e Zootecnia, Universidade de São Paulo, São Paulo, 2007.

MOTA, P.M.P.C. Tuberculose bovina: disgnóstico e controle. 2003. 48f. Tese (Doutorado em Ciência Animal) - Escola de Veterinária, Universidade Federal de Minas Gerais, Belo Horizonte, 2003.

PIGNATA, W.A.; ALVES, C.J.; AZEVEDO, S.S.; PINHEIRO, S.R.; VASCONCELLOS, S.A.; ALMEIDA, C.A.; DANTAS, A.F.M.; REMÍGIO, F.R. Tuberculose em caprinos no semi-árido brasileiro. Arquivos do Instituto Biológico, São Paulo, v.77, n.2, p. 339-342, 2010.

PINHEIRO, S.R.; ROXO, E.; ALMEIDA, C.A.S.; VASCONCELLOS, S.A.; SILVANTOS, M.C.C.; MAIORKA, P.C.; MELVILLE, A.M.P.; BENITES, N.R.; PAES, A.C. Surto de tuberculose em caprinos (Capra hircus). In: ENCONTRO NACIONAL DE PATOLOGIA VETERINÁRIA - ENAPAVE, 13., 2007, Mato Grosso do Sul. Anais. 2007. Disponível em: <www.eqrural.ufms.br/enapave>. Acesso em: 4 dez. 2009.

SILVA, P.E.G.; PINHEIRO, S.R.; LEAL, M.L.R.; BERTAGNON, H.G.; MOTA, P.M.P.C.; SINHORINI, I.L.; VASCONCELLOS, S.A.; BENESI, F.J. Teste de tuberculinização em caprinos (Capra hircus) experimentalmente sensibilizados. Ciência Rural, v.36, n.3, p.880-886, 2006.

SUTHER, B.E.; FRANTI, C.E.; PAGE, H.H. Evaluation of a comparative intradermal tuberculin test in California dairy cattle. American Journal of Veterinary Research, v.35, n.3, p.379-387, 1974.

VALENTE, L.C.M. Determinantes econômicos da sanidade bovina. 2009. 116f. Dissertação (Mestrado em Economia Aplicada) - Departamento de Economia, Universidade Federal de Viçosa, Viçosa, 2009.

ZAR, J.H. Bioestatistical analysis. 2.ed. Englewood Cliffs: Prentice-Hall, 1984. 450p.

Recebido em 4/4/11

Aceito em 16/5/12 